\title{
Perfil epidemiológico dos casos de afogamentos no norte do Brasil, com ênfase no estado do Pará de 2010 a 2019
}

Epidemiological profile of drowning cases in Northern Brazil, with an emphasis on the State of Pará from 2010 to 2019

Perfil epidemiológico de casos de ahogamiento en el norte de Brasil, con énfasis en el Estado de Pará de 2010 a 2019

\section{Resumo}

Introdução: Este trabalho teve o intuito de identificar o perfil epidemiológico das vítimas de afogamento do Estado do Pará entre o ano de 2010 a 2019. Objetivo: Traçar o perfil epidemiológico dos óbitos por afogamento no estado do Pará no período de 2010 a 2019. Métodos: Análise dos dados obtidos através da plataforma online do Departamento de Informática do Sistema Único de Saúde (DataSus). Resultados: No período, foram registrados 3.144 óbitos por afogamento e submersão acidentais, desses, $82,6 \%$ eram do sexo masculino e autodeclarados pardos (82,54\%). A faixa etária com maior número de casos foi de 20 a 29 anos seguida pela faixa etária de 1 a 4 anos. Os municípios que registraram maior número de mortes foram Belém, Marabá e Santarém. Conclusão: Há necessidade de maiores discussões sobre o assunto, pois dados sobre a caracterização do perfil epidemiológico das vítimas de afogamento 
permitirá ações preventivas específicas para determinadas faixas etárias em localidades e populações onde estes números são mais expressivos.

Palavras-chave: Afogamento; Mortalidade; Epidemiologia; Sistemas de informação.

\begin{abstract}
Introduction: This study aimed to identify the epidemiological profile of drowning victims in the State of Pará from 2010 to 2019. Objective: To trace the epidemiological profile of drowning deaths in the State of Pará from 2010 to 2019. Methods: Analysis of data obtained through the online platform of the Informatics Department of the Unified Health System (DataSus). Results: In the period, 3,144 deaths were registered due to accidental drowning and submersion, of which $82.6 \%$ were male and self-declared brown $(82.54 \%)$. The age group with the highest number of cases was 20 to 29 years old, followed by the age group 1 to 4 years old. The municipalities that registered the highest number of deaths were Belém, Marabá and Santarém. Conclusion: There is a need for further discussions on the subject, as data on the characterization of the epidemiological profile of drowning victims will allow specific preventive actions for certain age groups in locations and populations where these numbers are more expressive.

Keywords: Drowning; Mortality; Epidemiology; Information systems.
\end{abstract}

\title{
Resumen
}

Introducción: Este estudio tuvo como objetivo identificar el perfil epidemiológico de las víctimas de ahogamiento en el Estado de Pará de 2010 a 2019. Objetivo: Trazar el perfil epidemiológico de las muertes por ahogamiento en el Estado de Pará de 2010 a 2019. Métodos: Análisis de datos obtenidos mediante la plataforma en línea del Departamento de Informática del Sistema Único de Salud (DataSus). Resultados: En el período se registraron 3.144 muertes por ahogamiento accidental e inmersión, de las cuales el 82,6\% fueron varones y se declararon pardos (82,54\%). El grupo de edad con mayor número de casos fue el de 20 a 29 años, seguido del grupo de 1 a 4 años. Los municipios que registraron el mayor número de muertes fueron Belém, Marabá y Santarém. Conclusión: Es necesario profundizar en el debate sobre el tema, ya que los datos sobre la caracterización del perfil epidemiológico de las víctimas de ahogamiento permitirán acciones preventivas específicas para determinados grupos de edad en localizaciones y poblaciones donde estos números son más expresivos.

Palabras clave: Ahogamiento; Mortalidad; Epidemiología; Sistemas de información.

\section{Introdução}

O afogamento é a dificuldade respiratória causada pela entrada de líquido nas vias aéreas por submersão ou imersão nesse (OMS, 2014). Atualmente, ele está entre as três principais causas de morte fatal não intencional (Willcox-Pidgeon et al., 2016). Essa condição pode ser classificada como fatal, quando há afogamento e consequente morte por hipóxia tecidual, ou como não fatal, quando há interrupção do afogamento antes do óbito (Abelairas-Gómez et al., 2019).

Uma pessoa que está se afogando não consegue prender a respiração e manter a cabeça fora da água por muito tempo. Ao ser forçada a respirar, determinada quantidade de água é aspirada para as vias aéreas, e a tosse ocorrerá instantaneamente, como resposta reflexa, numa tentativa de proteger o organismo. A laringe pode sofrer espasmo (laringoespasmo), embora seja um evento raro (menos de 2\%), e logo essa sensação de sufocamento é aliviada pela hipóxia cerebral. Se não houver o resgate, maior quantidade de água continuará a ser aspirada, levando rapidamente o indivíduo à inconsciência e apneia. A taquicardia dará sequência à bradicardia, atividade elétrica sem pulso (AESP) e, finalmente, à perda completa do ritmo cardíaco e da atividade elétrica (assistolia). (Rocha \& Szpilman, 2019).

Em 2012, 91\% dos casos de morte por afogamento ocorreram nos países de baixa e média renda, evidenciando a prevalência em países emergentes. Soma-se a isso, o fato de existir subnotificação e falha no sistema coleta de informações de óbitos nesses países (Tyler et al., 2017; Peden et al., 2016).

Diversos fatores específicos colocam as pessoas em maior risco de incidentes por submersão, saber identificar esses fatores expande o nível de atenção e ajuda a produzir estratégias e políticas de prevenção para diminuir tais ocorrências, O principal fator de risco é a supervisão imprópria para lactantes e crianças, adolescentes e adultos é o comportamento de risco e o uso de drogas ou álcool. (PHTLS, 2017).

O Brasil, país em desenvolvimento e litorâneo, tem muitas praias e rios por toda extensão. A região Norte do país tem a maior mortalidade em comparação a outras regiões, já que segundo o SOBRASA (Sociedade Brasileira de Salvamento 
Aquático), 70\% dos óbitos por afogamento acontecem em rios e represas, que são abundantes na região citada. Em conformidade com esses dados, a região Norte apresenta um risco de morte por afogamento de 4,7 por 100.000 habitantes, enquanto que a região Sudeste tem um risco de morte por afogamento de 2 por 100.000 habitantes (SOBRASA, 2020).

Estudos da OMS sugerem que os homens têm taxas de mortalidade, por afogamento, maiores do que as mulheres devido a maior tempo exposto a ambientes aquáticos e por um comportamento de risco maior, como, por exemplo, nadar sozinho, o consumo de álcool antes de entrar na água e o uso de embarcações numa proporção maior do que as mulheres (Clarino apud Souza, 2007).

Destarte, o afogamento é umas das principais causas de morte em crianças, jovens e adultos jovens no Brasil, sendo que em 2018 foi a segunda maior causa de óbito entre crianças de 1 a 4 anos, e o maior risco de morte por afogamento está na faixa etária de 15 a 19 anos, tendo em vista que o comportamento de risco e a falta de supervisão são fatores que aumentam a probabilidade de ocorrer um afogamento. A época do ano e o horário também são aspectos relevantes no tema de óbitos por afogamento, por exemplo, mais de $65 \%$ dos óbitos por afogamento aconteceram em fins de semanas e feriados, e mais da metade ocorrem entre 10:00 e 14:00 horas, com essas informações é possível traçar medidas de prevenção que possam diminuir o risco do acontecimento de óbitos por afogamento (SOBRASA, 2020).

Dessa forma, apesar do afogamento ser uma das causas mais prevalentes de morte não intencional, há baixas taxas de financiamento para pesquisa e, consequentemente, escassez de informações na literatura médica, principalmente sobre países da América do Sul. Evidenciando, assim, uma grande discrepância entre a prevalência do assunto e a disponibilidade de informações sobre esse (Abelairas-Gómez et al., 2019).

O afogamento, da mesma forma que os demais acidentes, acontece inesperadamente na maior parte das vezes, provocando desconforto no âmbito familiar e social, apesar disso, a melhor forma de evitar, seria a prevenção através de medidas que possam antecipar sua instalação ou até mesmo minimizar os danos causados pelo evento. (Silva, 2014).

Por isso, tendo em vista que o afogamento se trata de um problema de saúde pública, reconhecer melhor esse problema pode auxiliar na redução desse tipo de acidente. Logo, o presente estudo possui como problemática central responder a pergunta: qual o perfil epidemiológico das vítimas de afogamento do Estado do Pará?

\section{Metodologia}

Trata-se de uma pesquisa descritiva, pois a concepção de Gil (1999) a mesma tem o objetivo de descrever as características de determinadas populações ou fenômenos ou estabelecimento de relações entre as variáveis. Uma de suas características mais importantes está na utilização de técnicas padronizadas de coleta de dados. Lima-Costa \& Barreto (2003), também afirmam que a epidemiologia descritiva pode fazer uso de dados secundários (dados pré-existentes de mortalidade e hospitalizações, por exemplo) e primários (dados coletados para o desenvolvimento do estudo). O estudo também pode ser classificado como quantitativo, pois se enquadra no conceito de Knechtel (2014), na qual a pesquisa tem por base a quantificação dos dados e buscará medir opiniões e informações utilizando os recursos da estatística, como a porcentagem, a média, o desvio-padrão. Tais dados serão apresentados em forma de tabelas, gráficos ou textos. Por isso, este estudo faz um levantamento dos casos de afogamento que ocorreram no Pará durante os anos de 2010 a 2019. Os dados foram obtidos por meio do Sistema de Informações Hospitalares do SUS (SIH/SUS), disponível no site do Departamento de Informática do Sistema Único de Saúde (DATASUS). Esses dados foram analisados e transformados em gráficos e tabelas com as devidas descrições, comparações e discussões, que ao final de todo processo de analise dos dados, deram subsídios teóricos para proposições e orientações a cerca do tema e de suas problemáticas. 


\subsection{Local de Estudo}

Segundo o IBGE (Instituto Brasileiro de Geografia e estatística), o Brasil é o maior país da América do Sul e apresenta proporções continentais, estende-se por uma área de $8.514 .876,599 \mathrm{~km}^{2}$. Ele é dividido em cinco regiões e 27 unidades federativas, seus Estados. O país é dividido em cinco regiões geográficas: Região Norte, Região Nordeste, Região Centro-Oeste, Região Sudeste e Região Sul.

A região Norte compreende sete estados: Acre, Amazonas, Amapá, Pará, Rondônia, Roraima e Tocantins. Juntos, eles abrangem área territorial de 3.851.281 km representando quase 50\% do território nacional. Dentro na região, o Estado do Pará é o segundo maior estado brasileiro em área com seus 1247,6 mil km² de extensão territorial, sendo o mais populoso da região norte com 8.602.865 habitantes. Fazendo fronteira com os estados Amapá, Maranhão, Tocantins, Mato Grosso e Roraima, e com os países Suriname e Guiana, sua capital é Belém. (IBGE, 2018).

\section{Resultados}

\subsection{Afogamentos no Brasil}

Entre os anos de 2010 e 2019, o Brasil registou, 5.218.685 notificações de morte por afogamento, o que permite dizer que quase 1429 morrem por ano por esta causa. De acordo com Dados do Sistema de internações Hospitalares do SUS, a Região Sudeste lidera este Ranking com 2.486.507 Registro, sendo responsável por 47,6\% dos casos de mortes. A região Nordeste também apresenta alto numero de óbitos e corresponde a 22,7\% dos registros. Dessa forma, Sudeste e Nordeste acumulam quase $70 \%$ das mortes registradas em todo o território nacional. Neste mesmo intervalo temporal, a Região Norte apresentou os números mais baixos de registros quando comparadas com as duas regiões supracitadas, (271.997 mortes), sendo a região com menos notificações segundo informações do SIH/SUS.

Os estados por Região com maiores números de mortes por afogamento foram: O estado de São Paulo, Região Sudeste, foi a que mais registrou caso de afogamento acidental, totalizando 1.323.536 registros. Em seguida, o Estado do Rio Grande do Sul, da Região Sul, com 403.889 óbitos. No nordeste, o Estado da Bahia registrou 307.393 mortes. Goiás foi o estado do Centro-oeste com maior numero de registo, 121.019 óbitos. Por fim, o estado do Pará registrou 119.564 mortes na Região Norte. Dessa forma, São Paulo, Rio Grande do Sul, Bahia, Goiás e Pará formam o conjunto dos estados com maior numero de mortes em cada região.

\subsection{Afogamentos na Região Norte}

Durante o período de 2010 a 2019, de acordo com os dados obtidos pelo SIH/SUS de Óbitos por residência segundo Região/Unidade da Federação foram registrados um total de 7.727 mortes por afogamento em toda região norte do país. O numero de mortes somente no Estado do Pará foi de 3.144 casos correspondendo. Este número corresponde a 40,69\%, o que coloca o estado em primeiro lugar em casos de mortes por afogamento, seguido pelo Estado do Amazonas com 27,44\% dos casos. Com isso, os dois estados concentram praticamente $68 \%$ dos registros de óbitos. O Estado de Roraima, por sua vez, apresentou o menor número ocorrências (274 registros), ou seja, corresponde a 3,55\% dos casos notificados.

Em relação ao numero de notificações por ano, 2014 foi o ano com mais registro de mortes. Neste ano foram notificados 850 casos, correspondendo a 11\% dos registros desde 2010. Além deste ano, 2015 também registou 846 casos, correspondendo assim a 10,95\% das notificações desse período. Partindo desses dados, a média de afogamentos na região norte durante os anos de 2010 a 2019 foi de 772,7 casos por ano. (Gráfico 1). 
Gráfico 1: Número de afogamentos por ano na Região Norte do Brasil.

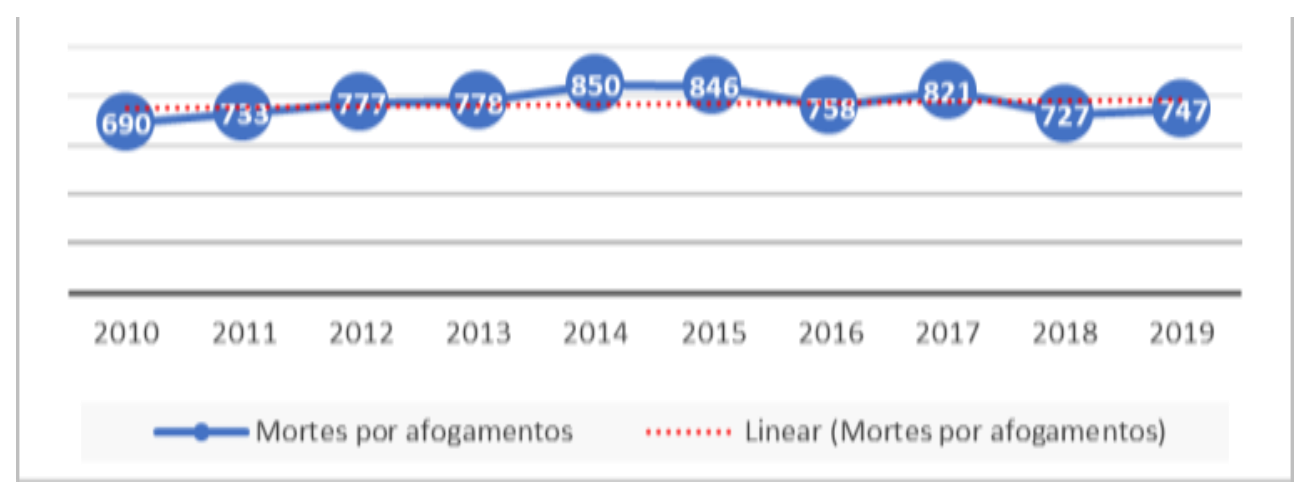

Fonte: Sistema de Informações Hospitalares do SUS.

Ainda de acordo com os dados do SIH/SUS, na Região Norte, as vítimas do sexo masculino $(\mathrm{n}=6.582)$ foram mais predominantes quando comparada ao sexo feminino $(n=1.135)$. A proporção de afogamento por sexo corresponde a quase 6:1, ou seja, $85,18 \%$ são do sexo masculino.

Quando se considera as notificações de acordo com a faixa etária percebe-se que a maior parte dos registros de indivíduos com idades entre 20 a 29 anos (n=1.289), ou seja, 16,68\% dos casos, seguida por indivíduos que possuíam de 30 a $39 \operatorname{anos}(n=1164)$ correspondendo a 15,06\% dos registros. Destaca-se ainda que, crianças de 1 a 4 anos formam o terceiro grupo mais atingido. Ao longo desse período foram registrados 1062 notificações, o que corresponde a 13,74\%. Dessa forma, na região norte, adultos de 20 a 39 anos e crianças de 1 a 4 anos correspondem aproximadamente $45 \%$ das mortes da Região.

Em relação aos registros de afogamentos de acordo com o mês de notificação observou-se que Julho, Agosto e setembro apresentaram respectivamente $768,748,729$ casos ao longo do período estudado, representado quase $30 \%$ dos casos notificados na série histórica. Percebe-se também que, dentre os três meses citados, o mês de junho apresenta maior incidência dos óbitos, diminuindo nos meses subsequentes. (Gráfico 2).

Gráfico 2: Mortes por afogamento de acordo o mês de notificação.

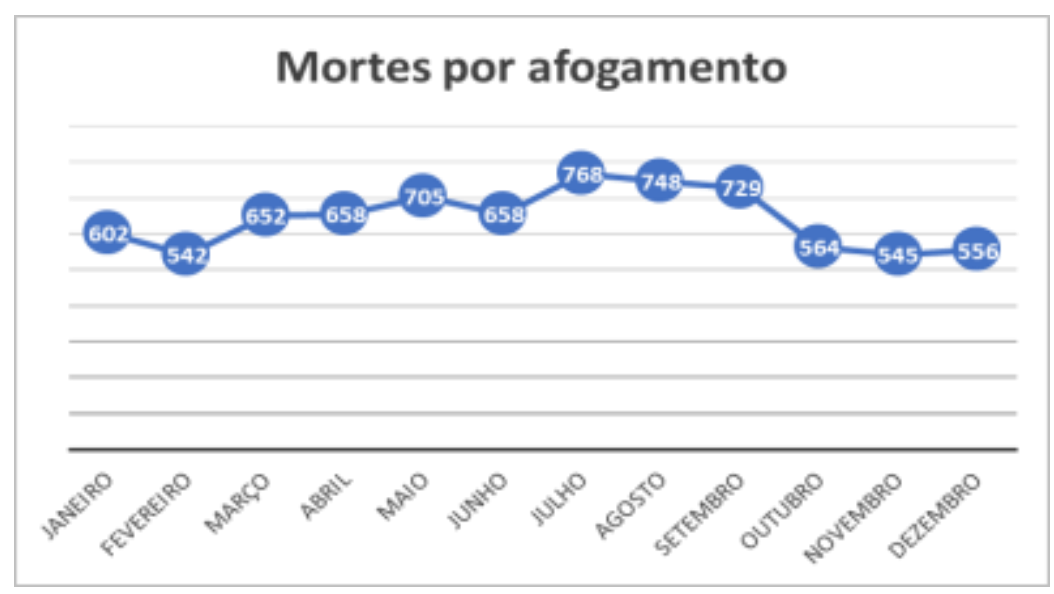

Fonte: Fonte: Sistema de Informações Hospitalares do SUS.

\subsection{Afogamentos no Pará}

Os dados coletados do Sistema de Informações Hospitalares do DATASUS registraram 3.144 óbitos por afogamento e submersão acidental no período entre 2010 a 2019 no Pará. O maior número de notificações foi no ano de 2014 em que fora apresentado 372 óbito, o que equivale a quase $12 \%$ de todos os casos do Estado. É possível perceber também que o ano de 
2018 apresentou o menor número de casos, 268 óbitos. Ao longo desse período o estado do Pará acumula uma média de morte de 314 por ano. (Gráfico 3)

Gráfico 3: Número de óbitos por afogamento no período de 2010-2019.

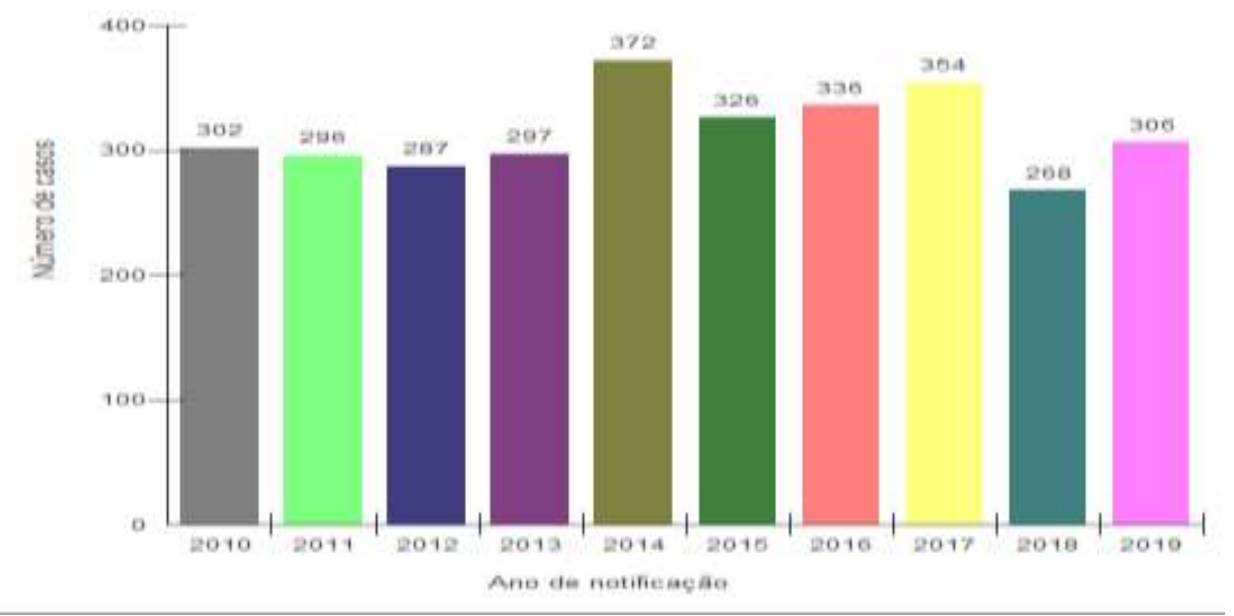

Fonte: Sistema de Informações Hospitalares do SUS.

Em relação a prevalência dos óbitos por afogamento segundo a faixa etária, o maior número de casos registrados está na faixa etária de 20 a 29 anos, enquanto o menor número de casos apresentados se encontra acima de 80 anos ou mais. É possível notar que, a partir da faixa etária de maior prevalência, ocorre um decrescimento no número de casos, ou seja, o numero de óbitos diminui de maneira inversamente proporcional à medida que a faixa etária aumenta. É importante destacar também, o alto numero de óbitos por afogamento em menores de 14 anos, principalmente com crianças na faixa etária entre 1 a 4 anos registrou 444 registros de mortes ente 2010 a 2019. (Gráfico 4)

Gráfico 4: Número de óbitos por afogamento segundo a faixa etária de 2010 a 2019.

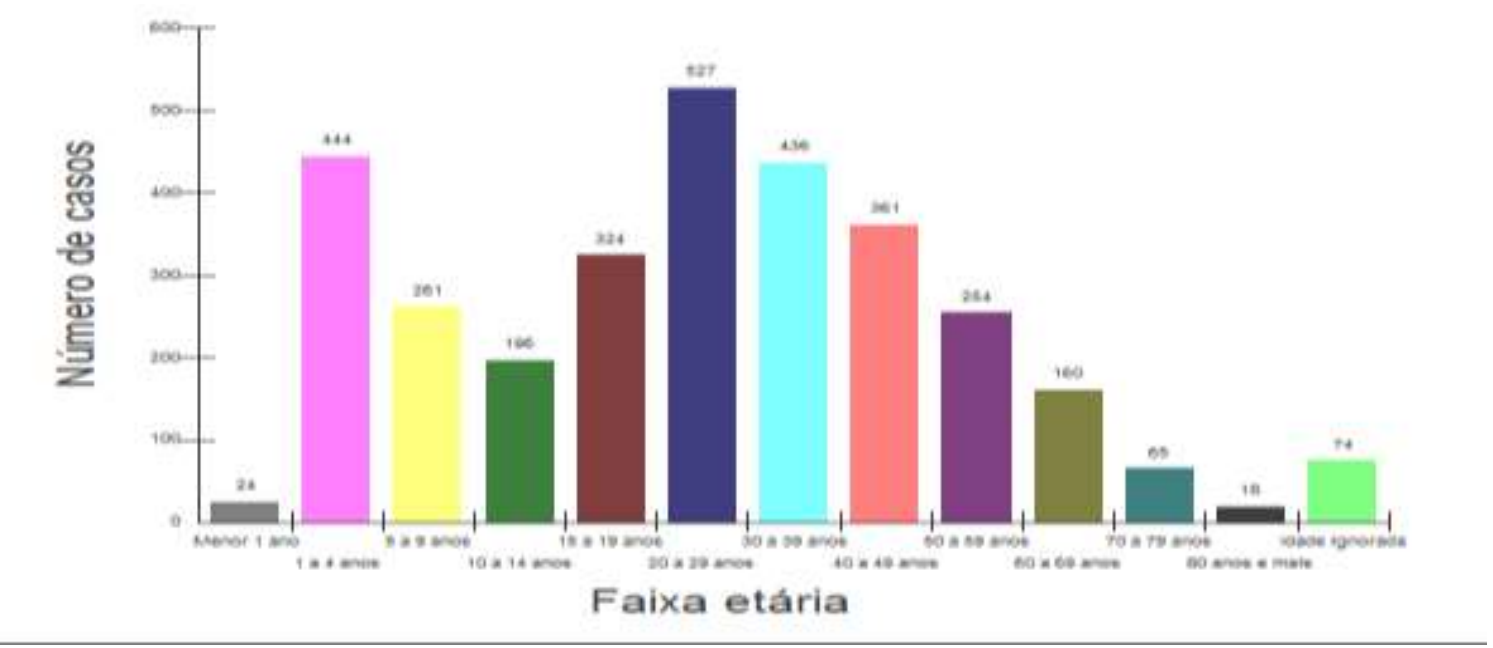

Fonte: Sistema de Informações Hospitalares do SUS.

Durante o período de 2010 a 2019, o óbito por afogamento para o sexo masculino foi o mais prevalente, cerca de $84,60 \%$ dos casos. Além disso, a escolaridade na maioria dos casos foi informada como ignorada (29,40\%). Os dados analisados apontam que a raça parda é a com mais registros de óbito por afogamento $(82,54 \%)$. 
Em relação ao sexo houve predominância do sexo masculino sobre o feminino com 2659 óbitos contra 482 casos respectivamente. Neste caso, o numero de mortes por afogamento do sexo masculino corresponde a $84,60 \%$ dos registros.

É importante destacar também a relação entre cor/etnia e o numero de registro de afogamentos no estado do Pará. Nesta relação prevaleceu consideravelmente a cor/etnia parda sobre todas as outras. Dados dos SIH/SUS registam que 82,54\% dos afogamentos são de pessoas Pardas. Os dados também mostram que os menores números de registros estão entre os declarados de cor Amarela e de etnia indígena equivalendo respectivamente $0,06 \%$ e $0,8 \%$ dos casos.

Dentro do Estado do Pará, 10 municípios, organizados em ordem decrescente, possuem os maiores registros de óbitos por afogamento acidentais: Belém, Marabá, Santarém, Altamira, Ananindeua, Parauapebas, Itaituba, Abaetetuba, Tucuruí e Breves. Esses municípios concentram aproximadamente 43\% dos registros de todo o estado. É importante destacar que os três municípios desta lista, Belém, Santarém e Marabá registraram 374, 186, 185 óbitos respectivamente durante o período de 2010 a 2019 concentrando, assim, cerca de 55\% dos casos registrados entre os 10 municípios listados com maiores números de mortes por afogamento.

\section{Discussão}

Mediante a análise feita no presente trabalho, observou-se um número significante alto de mortes no estado do Pará decorrentes de afogamentos e submersão acidental no intervalo de 2010 a 2019, com 3.144 falecimentos registrados somente no SIH/SUS. Enfatizando-se a prevalência de casos entre cidadãos do sexo masculino, pardos e nas faixas etárias de 20 a 29 anos e abaixo dos 14 anos de idade.

Primeiramente, pontua-se como um elemento crucial para situação analisada no Pará a disposição hidrográfica do estado, abundantemente cortada por rios afluentes do Rio Amazonas que constituem ferramenta importante para o comércio, turismo, economia e lazer da população (De castro, 2012). Nesse sentido, estudos conduzidos por Peden e al (2016) identificaram a distribuição e acesso de rios e lagos como fatores de risco para a criação de locais propícios para o acontecimento de episódios de afogamentos, uma vez que uma parcela deles não possuem postos de vigilância, avisos de perigo e são facilmente acessados em qualquer época do ano.

No que se refere ao maior acometimento de homens $(84,60 \%)$ em relação a mulheres $(15,40 \%)$, uma série de estudos a respeito do tema demonstraram resultados similares aos encontrados no estado do Pará (Moura 2010; Gomes; Biffi \& Ribeiro, 2017; Wang et al., 2019;). Dentre as principais causas apontadas, destaca-se a tendência dos homens a se arriscar mais em ambientes de risco para afogamentos, maior participação em atividades e empregos que envolvam a exposição aquática, superestimação de suas habilidades em natação, devido o consumo de drogas ou álcool e menor receio em relação a mulheres de se aventurar em localidades mais profundas ou desconhecidas (Howland et al., 1996; Koon et al., 2021).

Outro fator preponderante observado foi a maior taxa de mortes entre jovens e adolescentes, de 20 a 29 anos, vítimas de afogamento. Esses resultados se assemelham aos obtidos por Gomes et al (2017), onde as ocorrências e mortes em jovens de 20 a 29 anos também foram maiores, devido à falta de habilidade desse grupo em nado, imprudência e não supervisão adequada.

Além dos adolescentes e jovens afetados, outra faixa etária analisada como muito atingida por casos de afogamento no Pará foram crianças entre 1 a 4 anos, com 444 mortes no período de estudo. Segundo Peden et al. (2009) o afogamento está, atualmente, entre as 10 principais causas de óbitos infantis em todo mundo, afetando principalmente crianças com idade inferior a 5 anos de idade e sendo um grave problema em países em desenvolvimento como o Brasil. As mortes registradas nessa idade no estado podem ser resultado de uma série de fatores como é o caso vigilância inadequada por parte de pais e 
responsáveis para essas crianças, baixa habilidade de nado, maior facilidade de acesso a ambientes aquáticos como no caso das piscinas, exposição a baldes e banheiras com água e ausência de dispositivos de flutuação (Weiss et al., 2010).

Em relação à cor ou raça, a parda mostrou-se significativamente maior do que as outras etnias, representando 82,54\% dos afogamentos em território paraense. Esse resultado reforça estudos semelhantes feitos por Nogueira e colaboradores (2016) e corroborados por Xavier (2011), que elencaram a miscigenação ocorrida na população brasileira, ao longo do processo de formação histórica e geográfica do Brasil, como responsável pela predominância da cor/raça parda em grande parte do território nacional e, desse modo, a maior ocorrência de casos nesse grupo.

Ademais, é importante destacar que, mesmo com os altos números de afogamentos registrados no estado, ainda há uma subnotificação dos casos no Pará. A subnotificação ocorre principalmente pela inexistência de um sistema de dados unificados no Brasil, associado ao não preenchimento correto de boletins, especialmente no que se refere aos afogamentos não fatais, que são a maioria das ocorrências (Segundo; Sampaio, 2015).

Mediante esses aspectos, o presente trabalho buscou caracterizar o perfil epidemiológico dos casos de afogamentos no Pará para fornecer subsídios teóricos para a elaboração, por parte das autoridades competentes, de ações que visem mitigar a ocorrência desses episódios. Aconselha-se o planejamento de campanhas educativas e de prevenção a afogamentos em todo o território do estado, em especial voltadas para o público jovem e pais, haja vista a alta incidência de afogamentos na população abaixo dos 29 anos. Além disso, recomenda-se a criação de barreiras de acesso a locais com alto risco de afogamentos e o melhor registro de dados de forma unificada e ampla dos episódios (Leavy et al., 2016).

\section{Conclusão}

O numero de casos de afogamento no Estado do Para, obtidos pelo Sistema de Informação do DATASUS, foi de 3.144 óbitos e possibilitou identificar prevalência de mortalidade por afogamento entre homens $(84,6 \%)$, principalmente entre indivíduos da cor de pele parda $(82,54 \%)$. O ano de 2014 registrou o maior numero de notificações de afogamento. Nesse intervalo temporal o Estado do Pará registrou em média 314 óbitos anuais. As faixas etárias de maior prevalência de acidentes por afogamento são jovens entre 20-29 anos e crianças entre 1-4 anos de idade. Na analise observou que Belém, Marabá e Santarém concentram a maior quantidade de notificações por afogamento em todo o Estado.

Este trabalho retrata a necessidade de discussão sobre o tema, pois caracterizar o perfil epidemiológico das vítimas de afogamento permite ações preventivas específicas que visem diminuição da mortalidade por este tipo de agravo além de ações de fiscalização em localidades e populações onde estes números são mais expressivos. Por isso, Sugere-se para futuros trabalhos verificar a existência e/ou desempenho das equipes de atendimento inicial as vitimas de afogamento, principalmente nos municípios paraenses com maiores incidências, a fim de que, à partir dos resultados obtidos, seja possível planejar ações que visem diminuir o número de vitimas.

\section{Referências}

Abelairas-Gómez, C., Tipton, M.J., González-Salvado, V. \& Bierens, J.J.L.M. (2019) Drowning: epidemiology, prevention, pathophysiology, resuscitation, and hospital treatment. Emergencias. Mar; 31(4):270-280. English, Spanish. PMID: 31347808. https://pubmed.ncbi.nlm.nih.gov/31347808/

Bierens, J. J., Lunetta, P., Tipton, M.J. \& Warner, D. S. (2016) Physiology Of Drowning: A Review. Physiology (Bethesda). Mar; $31(2)$ : 147-66. https://journals.physiology.org/doi/full/10.1152/physiol.00002.2015

De castro,V.V. (2012). Fatores que influenciam a gestão de recursos hídricos de áreas edificadas: o caso da região amazônica. Tese de Doutorado (Engenharia Civil) - Universidade Federal Fluminense, Niterói. http://ole.uff.br/wp-content/uploads/sites/461/2018/10/tese_formatada.pdf.

Gil, A. C. (1999). Métodos e técnicas de pesquisa social. 5.ed. São Paulo: Atlas. https://ayanrafael.files.wordpress.com/2011/08/gil-a-c-mc3a9todos-etc3a9cnicas-de-pesquisa-social.pdf 
Gomes, G.A., Biffi, D., \& Ribeiro, V.R. (2017) Perfil epidemiológico das vítimas de afogamento do estado do rio grande do sul. Revista Perspectiva: Ciência e Saúde, v. 2, n. 2. http://sys.facos.edu.br/ojs/index.php/perspectiva/article/view/110/95.

Howland, J. et al.(1996) Why are most drowning victims men? Sex differences in aquatic skills and behaviors. American journal of public health, v. 86, n. 1, p. 93-96. https://ajph.aphapublications.org/doi/pdf/10.2105/AJPH.86.1.93.

IBGE - INSTITUTO BRASILEIRO DE GEOGRAFIA E ESTATÍSTICA (BR). (2018). Cidades e Estados. Rio de Janeiro: IBGE.

Knechtel, M.R. (2014). Metodologia da pesquisa em educação: uma abordagem teórico-prática dialogada. Curitiba: Intersaberes.

Koon, W. et al. (2021) Coastal drowning: A scoping review of burden, risk factors, and prevention strategies. PLoS one, v. 16, n. 2 , p. e0246034. https://journals.plos.org/plosone/article?id=10.1371/journal.pone.0246034.

Leavy, J.E. et al. (2016). A review of drowning prevention interventions for children and young people in high, low and middle income countries. Journal of community health, v. 41, n. 2, p. 424-441. https://link.springer.com/article/10.1007\%2Fs10900-015-0105-2.

Lima-costa, M.F., \& Barreto, S.M. (2003). Tipos de estudos epidemiológicos: conceitos básicos e aplicações na área do envelhecimento. Epidemiol. Serv. Saúde, Brasília, v. 12, n. 4, p. 189-201, dez. http://scielo.iec.gov.br/scielo.php?script=sci_arttext\&pid=S1679-49742003000400003\&lng=pt\&nrm=iso.

MINISTÉRIO DA SAÚDE (MS). Acidentes por afogamento. http://bvsms.saude.gov.br/dicas-em-saude/2401-acidentes-por-afogamento.

Mott,T.F., \& Latimer, K.M. (2016) Prevention and Treatment of Drowning. Am Fam Physician. Abr. 1; 93(7): $576-82$. https://www.aafp.org/afp/2016/0401/p576.html

Moura, A.C. (2010). Perfil epidemiológico dos óbitos por afogamento no estado de Alagoas-Brasil no período de 2000-2008. Dissertação de Mestrado (Saúde e Ambiente) - Universidade Tiradentes, Aracajú. http://openrit.grupotiradentes.com:8080/xmlui/handle/set/2967.

Nogueira, C.M. et al. (2016). Mortalidade por afogamento em crianças menores de 5 anos no Brasil: 2001 a 2010. Rev. Baiana Saúde Pública, v40. nº3. a1701. https://rbsp.sesab.ba.gov.br/index.php/rbsp/article/view/1701/2062.

Organização Mundial da Saúde (OMS). (2014). Informação mundial sobre afogamento: prevenção - o primeiro elo da cadeia da sobrevivência. In: global report on drowning. [S. 1.]. https://apps.who.int/iris/bitstream/handle/10665/143893/9789241564786-por.pdf?sequence=5\&isAllowed=y

Peden, A.E., Franklin, R.C., \& Legga, T.P.A. (2016) Fatal river drowning: the identification of research gaps through a systematic literature review. Inj Prev. Jun; 22(3): 202-9. https://injuryprevention.bmj.com/content/injuryprev/22/3/202.full.pdf.

Peden, M. et al. (2009) World report on child injury prevention. Geneva: World Health Organization, https://www.ncbi.nlm.nih.gov/books/NBK310641/

PHTLS N. (2011) Atendimento pré-hospitalar ao traumatizado. Rio de Janeiro/RJ, Elsevier ;7: 53-78.

Rocha, M. P., \& Szpilman, S. D. Afogamentos (Capítulo de livro). http://www.szpilman.com/new_szpilman/szpilman/ARTIGOS/Cap\%203\%20\%20Afogamento\%20\%2005-11-15_szpilman.pdf

Segundo, A.S.S., \& Sampaio, M.C. (2015). Perfil epidemiológico dos afogamentos em praias de Salvador, Bahia, 2012. Epidemiologia e Serviços de Saúde, v. 24, p. 31-38. https://www.scielosp.org/pdf/ress/2015.v24n1/31-38/pt.

Silva, P.W. (2014). Prevenção de Afogamentos: Aulas de Natação, o campo Propicio para aplicar as medidas preventivas e de sobrevivência aquática minimizando os possíveis danos causados por este acidente. UEPB, Campus Campina Grande, Paraíba, 9-22.

http://dspace.bc.uepb.edu.br/jspui/handle/123456789/3405

Souza, D.C. (2007). Prevenção: da importância à prática no Salvamento Aquático. São José. https://www.sobrasa.org/prevencao-da-importancia-a-pratica-desalvamento-aquatico-monografia/

Szpilman, D. \& diretoria Sobrasa (2018). Afogamento - Boletim epidemiológico no Brasil 2020. Sociedade Brasileira de Salvamento Aquático SOBRASA Disponível em: http://www.sobrasa.org

Tyler, M.D, Richards, D. B., Reske-nielsen, C., Saghafi, O., Morse, E.A., Carey, R., \& Jacquet, G.A. (2017). The epidemiology of drowning in low- and middle-income countries: a systematic review. BMC Public Health. May 8;17(1):413. https://pubmed.ncbi.nlm.nih.gov/28482868/

Wang, L. et al. (2019). Unintentional drowning mortality in China, 2006-2013. Injury prevention, v. 25, n. 1, p. 47-51.

https://pubmed.ncbi.nlm.nih.gov/29691315/

Weiss, J. et al. (2010). Prevention of drowning. Pediatrics, v. 126, n. 1, p. e253-e262.

https://pediatrics.aappublications.org/content/pediatrics/126/1/e253.full.pdf.

Willcox-pidgeon, S.M., Franklin, R.C., Leggat, P.A., \& Devine, S. (2020). Identifying a gap in drowning prevention: high-risk populations. Injury Prevetion Jun; 26(3): 279-288. https://onlinelibrary.wiley.com/doi/10.1111/1753-6405.13102

Xavier, E.L.S.(2011). Perfil epidemiológico dos afogamentos fatais em crianças no Distrito Federal. Monografia (Pediatria) - Hospital Regional da Asa Sul, Brasília. file:///C:/Users/ferna/Downloads/Afogamentos_fatais_DF.pdf. 